\title{
A phase III double-blind placebo-controlled randomized study of dexamphetamine sulfate for fatigue in patients suffering from primary brain tumors
}

Florence Laigle-Donadey (1), François Ducray (2), Matthieu Boone (3), Hassimiou Diallo (4), Carole Ramirez (5), Olivier Chinot (6), Khê Hoang-Xuan (1), Damien Ricard (7), Jean-Yves Delattre (1)

(1) Service de Neurologie Mazarin, UPMC, APHP, GH Pitié-Salpêtrière, Paris, France, (2) Service de Neurologie, Hôpital Pierre Wertheimer, CHU de Lyon, Bron, France, (3) Service de Neuro-oncologie, CHU d'Amiens, France, (4) Unité de Recherche Clinique, APHP, GH Pitié-Salpêtrière, Paris, France, (5) Service de Neurochirurgie, hôpital $R$ Salengro, CHRU de Lille, France, (6) Service d'Oncologie, CHU de la Timone, Marseille, France, (7) Service de Neurologie, Hôpital d'Instruction des Armées de Percy, Clamart, France

Many primary brain tumor (PBT) patients complain of severe fatigue, which contributes to decreased quality of life (QOL).

\section{Objective:}

We hypothesized that dexamphetamine sulfate, a psychostimulating drug, could result in reduced fatigue in PBT patients.

\section{Methods:}

A phase III, multi-institutional, placebo-controlled randomized trial assessed the efficacy and tolerance of dexamphetamine administered up to 30 $\mathrm{mg} /$ day during $\mathbf{3}$ months in PBT patients with non progressive disease.

Patients should complain of severe fatigue, defined by a Multidimensional Fatigue Inventory (MFI) score $\geq 60$, and should not suffer from depression. Evaluation included treatment effect on fatigue (primary outcome).

We also studied as secondary outcomes anxiety and depression (Hospital Anxiety and Depression Scale), affectivity and fatigue (Norris Analogic Visual scale), Quality Of Life (EORTC QLQ C30 and Brain Module 20), cognitive functions, such as memory, speech, executive functions and apathy (Mattis dementia rating scale, Verbal Fluency Category, Trail Making Test Parts $A$ and $B$, Wisconsin Card Sorting Test, Marin apathy scale), as well as tolerance.

Conclusion: Dexamphetamine up to $30 \mathrm{mg} /$ day, is well tolerated in non progressive PBT patients suffering from severe fatigue, but does not provide a significant benefit on fatigue, anxiety, depression, cognition, and QOL.

\section{Results:}

From April 2013 to November 2016, a total of fourtysix patients from 7 different French centers were enrolled and randomized (23 in the intervention arm (IA) and 23 in the placebo arm (PA)), with 41 (89\%) retained for analysis:

Mean age: 57 years (IA)/51 years (PA)

Histology:

- mainly gliomas 17/22 (77\%)(IA)/17/19 (89\%) (PA);

- others : CNS lymphomas (4 cases (3IA+1PA)), medulloblastomas ( 3 cases (2IA+1PA))

Treatment: a maximal dose of $30 \mathrm{mg} /$ day of dexamphetamine sulfate, reached after 2 steps of 10 days of escalating dose (10 and $20 \mathrm{mg}$ ) in case of good tolerance.

Toxicity: Tolerance was generally good, with no treatment related death and no grade 4 toxicities. However, patients in the IA complained more frequently about psychiatric side effects (mostly hyperactivity, anxiety, sleep disorder, irritability...) than the patients in the PA $(p=0,018)$.

Efficacy: There were no statistically significant differences at 3 months between the dexamphetamine and placebo subgroups in the MFI 20 score (Figure).

\section{MFI 20 score variation between J0 and Month 3}

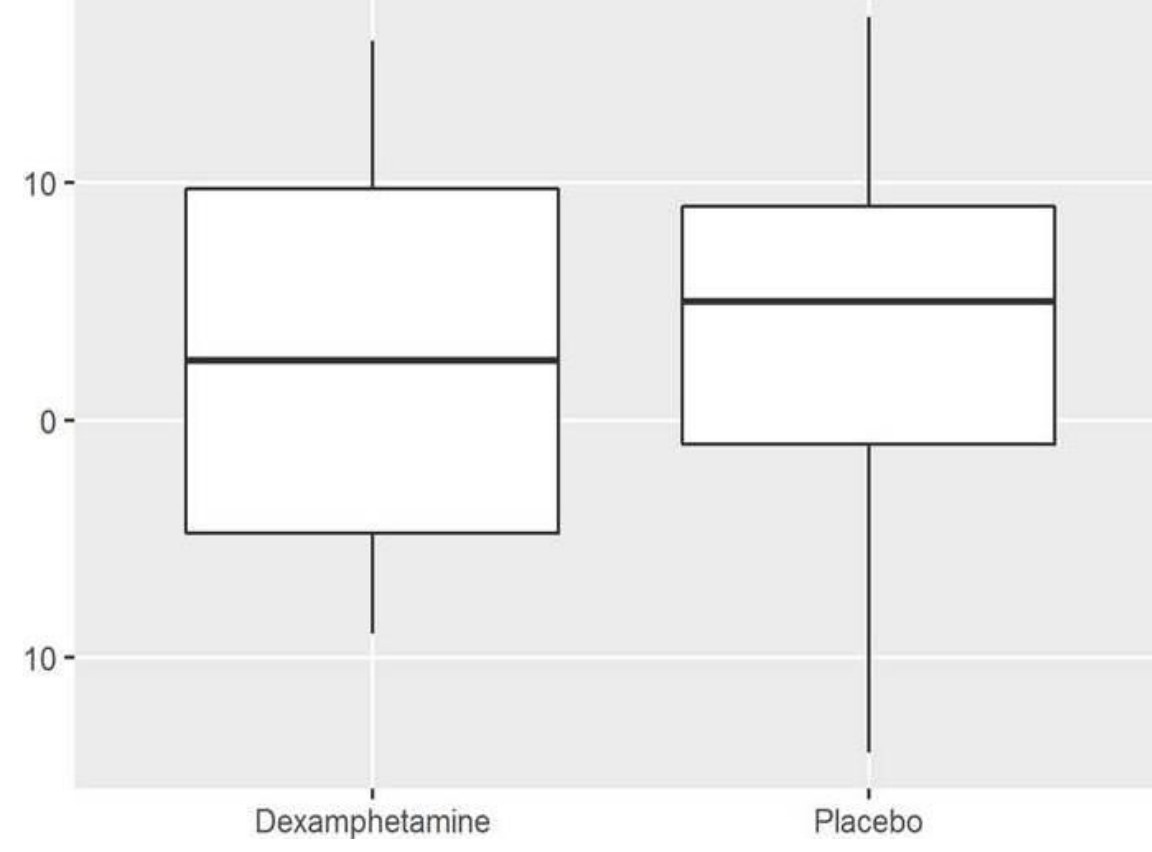

- There were also no statistically significant differences in any secondary outcomes : fatigue according to Norris scale, affectivity, anxiety, depression and cognitive functions. 